http://dx.doi.org/10.35381/i.p.v3i5.1368

\title{
Estudio etnobotánico y fitoquímico para la promoción del uso medicinal del dividivi (Caesalpinia coriaria (Jacq.) Willd.)
}

\section{Ethnobotanic and phytochemical study for the promotion of the medicinal use of dividivi (Caesalpinia coriaria (Jacq.) Willd.)}

\author{
Betsay María Toyo-Fernández \\ btoyofernandez@gmail.com \\ Universidad Nacional Experimental Francisco de Miranda, Santa Ana de Coro, Falcón \\ Venezuela \\ https://orcid.org/0000-0001-9679-747X
}

Recibido: 05 de abril de 2019

Revisado: 17 de mayo de 2019

Aprobado: 20 de junio de 2020

Publicado: 31 de julio de 2020 
INGENIUM ET POTENTIA

Revista Electrónica Multidisciplinaria de Ciencias Básicas, Ingeniería y Arquitectura
Año III. Vol III. N5. Julio - Diciembre. 2021
Hecho el depósito de Ley: FA2019000052
FUNDACIÓN KOINONIA (F.K).
Santa Ana de Coro, Venezuela.
Betsay María Toyo-Fernández

\title{
RESUMEN
}

Se proyecta una investigación mixta para evaluar desde el punto de vista etnobotánico y fitoquímico los frutos del dividivi en Carazao, estado Falcón, Venezuela como contribución al patrimonio ecológico, cultural y saludable de la región. A través del método etnográfico serán descritas las experiencias etnomedicinales de informantes claves de la comunidad. Cuantitativamente, la importancia cultural de la especie será determinada por el índice de Friedman, índice de valor de uso y consenso del uso. Para el estudio fitoquímico se prepararán extractos al 10\% P/V usando cuatro sistemas: uno acuoso y tres etanol-agua a los que se aplicarán los métodos de extracción: soxhlet, maceración pasiva, infusión y decocción. Posteriormente, se aplicarán los métodos de follinciocalteu y DPPH, para determinar compuestos fenólicos y actividad antioxidante. Los estudios etnobotánicos desde un enfoque interdisciplinario contribuyen a la búsqueda de nuevos agentes fitoterapéuticos y al desarrollo sostenible de las comunidades rurales portadoras del conocimiento tradicional.

Descriptores: Tecnología química; biotecnología; ciencias de la vida. (Palabras tomadas de Tesauro UNESCO).

\begin{abstract}
A mixed investigation is projected to evaluate from the ethnobotanical and phytochemical point of view the fruits of the dividivi in Carazao, Falcón state, Venezuela as a contribution to the ecological, cultural and healthy heritage of the region. Through the ethnographic method, the ethnomedicinal experiences of key informants from the community will be described. Quantitatively, the cultural importance of the species will be determined by the Friedman index, use value index and consensus of use. For the phytochemical study, extracts will be prepared at $10 \% \mathrm{~W} / \mathrm{V}$ using four systems: one aqueous and three ethanolwater to which the extraction methods will be applied: soxhlet, passive maceration, infusion and decoction. Subsequently, the follinciocalteu and DPPH methods will be applied to determine phenolic compounds and antioxidant activity. Ethnobotanical studies from an interdisciplinary approach accept the search for new phytotherapeutic agents and the sustainable development of rural communities that carry traditional knowledge.
\end{abstract}

Descriptors: Chemical technology; biotechnology; life sciences. (Words taken from UNESCO Thesaurus). 


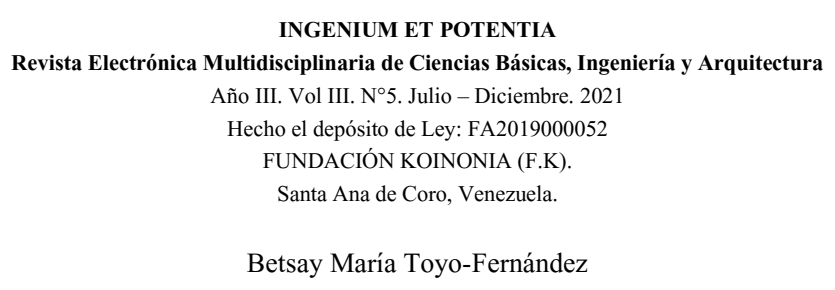

\section{INTRODUCCIÓN}

La necesidad de satisfacer el acceso universal a la salud ha llevado a organismos internacionales como la Organización Mundial de la Salud a fomentar el respeto y reconsideración de las prácticas tradicionales desarrolladas por cada cultura y región para promover, conservar y recuperar la salud. El conocimiento etnobotánico ha servido como facilitador en el proceso de descubrimiento de compuestos químicos con propiedades bioactivas que puedan convertirse en fármacos necesarios para el bienestar humano (Vargas y Lobato, 2018).

Aunado a esto, Adams et al., (2009), refieren que los estudios etnobotánicos son la base para el desarrollo de programas para más conocimientos sobre el uso de la medicina tradicional, enriqueciendo la herencia cultural y el uso adecuado de plantas medicinales en comunidades. Es por esto que, como cita Hernández et al., (2012) la fitoterapia avizora un futuro prometedor debido al aumento de la demanda actual de la población mundial como tratamiento tradicional por el envejecimiento poblacional y el consiguiente aumento de la incidencia de enfermedades.

Obregón (2012) (citado por Rodríguez, 2014), relata que los estudios etnobotánicos en comunidades son los primeros pasos en la aplicación terapéutica de los productos de origen vegetal para la prevención y tratamiento de las enfermedades. De acuerdo con Rosas (2015), el registro de los saberes tradicionales sobre la flora medicinal permite por un lado la conservación de este vasto conocimiento, pero además provee información fundamental para la selección de especies a la investigación farmacéutica. Va más allá de la simple acción de enlistar las plantas medicinales, sus usos y forma de preparación, es documentar cultural, ecológica, fitoquímica y farmacológicamente las plantas con uso terapéutico; lo cual sienta las bases para la elaboración de estrategias de aprovechamiento y el salvaguarda intelectual de estos saberes.

El valor agregado que proveen los métodos rigurosos de análisis fitoquímico al conocimiento etnobotánico se resume en la determinación precisa de las sustancias bioactivas responsables de las propiedades que el conocimiento tradicional les asigna 


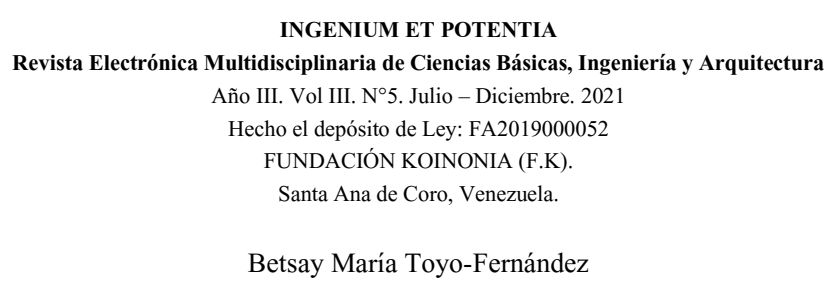

desde su experiencia; a fin de valorizar tanto a los conocimientos como a las especies y estimar científicamente el valor de uso de las plantas (Torres y Ganoza, 2017).

En este sentido, la búsqueda de plantas que contengan compuestos fenólicos se ha vuelto cada vez más relevante, ya que éstos tienen un efecto beneficioso en la prevención de enfermedades, pues la mayoría de ellos protegen del daño producido por los radicales libres causantes del estrés oxidativo. Esta búsqueda al azar puede ser muy exhaustiva y ocupar mucho tiempo; por esta razón, se recurre al conocimiento etnobotánico como fuente primaria para la selección plantas con potencial fitoterapéutico que puedan ser analizadas en los laboratorios.

En este orden de ideas, uno de los árboles más comunes en las zonas semiáridas del estado Falcón es el dividivi (Caesalpinia coriaria (Jacq.) Willd.), su distribución y abundancia es muy extensa; además, su productividad máxima alcanza hasta $800 \mathrm{Kg}$ de frutos por árbol por año. Dichos frutos, fueron producto venezolano de exportación durante casi dos siglos utilizándose como fuente de taninos para el curtido de pieles y en el proceso de recuperación de crudos (Díaz, 2001). El conocimiento etnobotánico del dividivi en el estado Falcón no ha sido documentado, distintos estudios desarrollados en Colombia y algunos estados de Venezuela han referenciado que la decocción de los frutos secos se usan para curar las amigdalitis, aftas, diarreas y dolor de estómago; en forma de baños es usado para problemas de hemorroides, prolaxos, enfermedades de la piel, heridas y quemaduras (Bello, 2017; Jaramillo et al., 2014; Rosado y Moreno, 2010; Sarmiento, Espitia y López, 2017).

Sin embrago, a pesar de que es conocido el alto contenido de taninos hidrolizables que presentan los frutos del dividivi (Álvarez y Lock,1992), no se han realizado en el estado Falcón análisis fitoquímicos que determinen específicamente su contenido fenólico y capacidad antioxidante; para definir con precisión la base de su acción terapéutica. Estudios internacionales en extractos de Caesalpinia coriaria (Jacq.) Willd revelan que su contenido fenólico y capacidad antioxidante es considerable, superando a otras plantas 


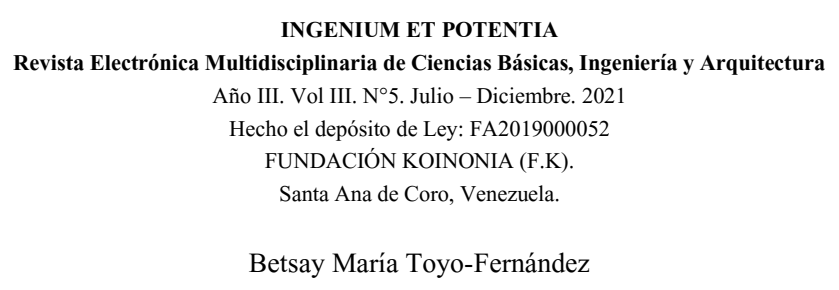

medicinales (Amudha y Shanthi, 2011) y que además presenta un potencial antineoplásico (Sánchez et al., 2017).

Estos precedentes se convierten en la idea impulsora para desarrollar el presente proyecto en la comunidad de Carazao del Municipio Miranda, Estado Falcón, desde una visión etnográfica, a fin de "describir lo que la gente hace desde la perspectiva de la gente" (Restrepo, 2016), con la finalidad de rescatar los conocimientos ancestrales de la comunidad que enriquezcan su herencia cultural y el uso adecuado del dividivi como planta medicinal, lo que adicionalmente proporciona información valiosa de su potencial fitoterapéutico que debe ser determinado fitoquímicamente, atendiendo al "desarrollo de la industria farmacéutica con respeto y aprovechamiento de la biodiversidad para la atención y protección de la población" (Plan de la Patria 2019-2025, p.50).

Dadas las consideraciones que anteceden, el presente estudio tiene como objetivo realizar una revisión documental que considere todos los aspectos necesarios para evaluar desde el punto de vista etnobotánico y fitoquímico los frutos secos del dividivi (Caesalpinia coriaria (Jacq.) Willd.) en la comunidad de Carazao del estado Falcón como contribución al patrimonio ecológico, cultural y saludable de la región, lo cual busca posicionar a esta comunidad como eslabón inicial de la cadena de investigación bioprospectiva, valorando los saberes tradicionales.

\section{Etnobotánica}

La investigación sobre el uso de plantas medicinales forma parte de la etnobotánica, la cual es una rama de la etnobiología que se encarga de estudiar la relación directa entre los grupos humanos y los vegetales, esto incluye plantas alimenticias, medicinales, ornamentales, artesanales, entre otras (Casale et al., 1999; Gómez, 2002; Martin, 2001, citados por Bello, 2017). Conceptualmente la etnobotánica rescata y revaloriza, desde una perspectiva holística, la historia de los vegetales en las sociedades a través del tiempo y del espacio. Esta relación sociedad-planta es siempre dinámica, por parte de la sociedad intervienen fenómenos culturales, ideológicos, políticos y económicos y por 


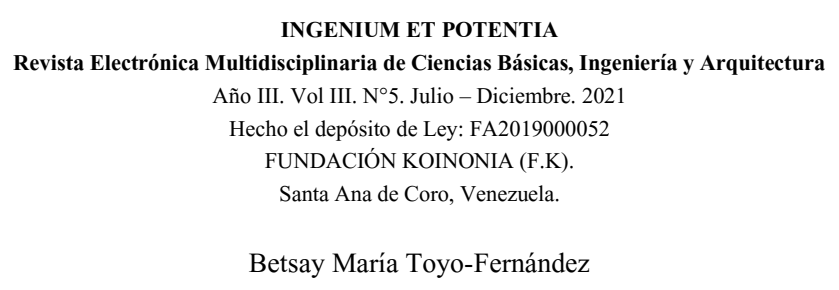

parte de los especímenes, el ambiente con sus biomasas y sus floras (Gispert et al., 2008, citado por Rodríguez, 2014).

Hay que destacar que las investigaciones etnobotánicas tienen varios aspectos de vital importancia: la protección de las especies en peligro de extinción, el rescate de los conocimientos sobre los vegetales y sus propiedades que poseeen las diferentes culturas del mundo, la domesticación de nuevas plantas utiles en términos más amplios y la conservación del plasma genético de plantas económicamente prometedoras (Waizel, 2010, citado por Rodríguez, 2014).

Para lograr recabar esta información sobre el conocimiento de las comunidades se emplean cuestionarios o entrevistas etnofarmacológicas participativas, cuyo punto de partida no son las plantas sino los síntomas o problemas de salud tal y como son percibidos por los grupos humanos que colaboran respondiendo estos instrumentos. El análisis casuístico de cada uso, de los antecedentes bibliográficos de la afección de salud y de la planta en cuestión han derivado en recomendaciones de investigaciones de laboratorio (químicas, farmacéuticas, y clínicas), cuyos resultados han sido revisados por el colectivo científico, concluyendo en recomendaciones específicas (Gómez-Beloz, 2002; Ploetz y Orr, 2004, citados por Dávila et al., 2016).

\section{Estudios etnobotánicos interdisciplinarios}

Según Bermúdez et al., (2005), un proyecto etnobotánico interdisciplinario, dentro de una comunidad o región determinada, constaría de las siguientes cuatro fases:

a- Documentación del conocimiento tradicional: La documentación del conocimiento tradicional sobre las plantas medicinales utilizadas en el contexto cultural seleccionado constituye la primera fase de cualquier proyecto y está dirigida a registrar los usos tradicionales de las plantas medicinales dentro de un contexto cultural determinado. Previamente, los investigadores deben negociar con la comunidad a estudiar su autorización para desarrollar el proyecto y los mecanismos para compensar la participación de los informantes locales. 


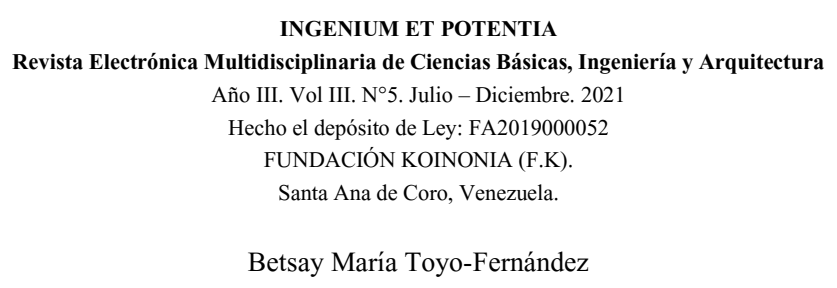

b- Determinaciones cuantitativas: Comprende la determinación cuantitativa de la importancia relativa, distribución y abundancia de las plantas medicinales usadas, las estrategias etnoecológicas para el aprovechamiento de tales recursos locales, así como del patrón de variación del conocimiento tradicional en la población seleccionada para el estudio. Aunque los índices constituyen una técnica que evalúa solo uno de los atributos relacionados con el conocimiento tradicional y estadísticamente son de tipo paramétrico, sujetos a aquellos factores que afectan las medidas de tendencia central, pueden servir de referencia en la selección de especies para evaluaciones químicas y farmacológicas posteriores.

c- Evaluación fitoquímica, farmacológica y toxicológica: A partir de la información registrada en las dos primeras fases de la investigación, se seleccionan aquellas especies de plantas medicinales con mayor importancia cultural para el grupo humano estudiado y luego se evalúan experimentalmente para conocer su composición química, estimar el potencial citotóxico de sus extractos y determinar si su actividad farmacológica se corresponde con los usos atribuidos por los informantes.

d- Desarrollo de mecanismos para compensación a la comunidad: Es necesario desarrollar mecanismos para compensar a la comunidad por su participación en la investigación, reconocer los derechos de propiedad intelectual del conocimiento tradicional sobre plantas medicinales y la conservación de los ecosistemas fuente y las especies útiles. Esto se traduce en ofrecer a las comunidades locales los resultados y conclusiones de las investigaciones, fortalecer los sistemas tradicionales de producción agrícola, promover el uso racional de las plantas en el cuidado de la salud y fomentar el conocimiento ecológico tradicional. Aunque la escala y objetivos de cada proyecto varían, todos deben tener en común el ser impulsados por el entusiasmo de los investigadores y la gente de las comunidades, quienes trabajando juntos pueden mejorar las condiciones locales. 


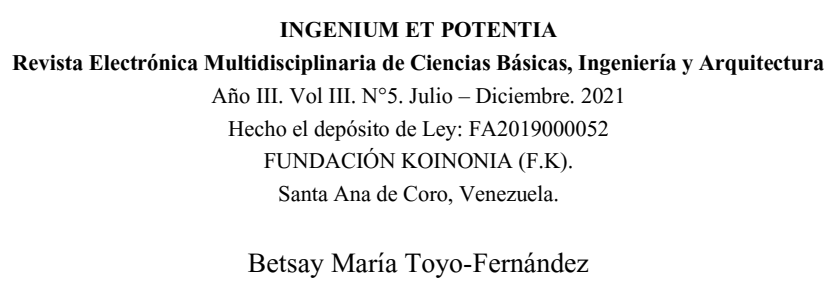

\section{Dividivi (Caesalpinia coriaria (Jacq.) Willd.)}

Árbol perteneciente a la familia botánica FABACEAE, el tallo tiene de 3 a $10 \mathrm{~m}$ de altura, crispado-pubescente a hirtuloso. Hojas en pinnas de 3 a 10 pares, de 5 a $12 \mathrm{~cm}$ de largo, cada una con 10 a 25 pares de folíolos de 4 a $8 \mathrm{~mm}$ de largo y $2 \mathrm{~mm}$ de ancho; ápice redondeado; comúnmente con una pinna terminal.

Inflorescencias con 3 a $6 \mathrm{~cm}$ de longitud, panículas individuales racemosas 1 a $2 \mathrm{~cm}$ de largo, con 15 a 20 flores; pétalos color crema amarillento a blanco-verdosos, carnosos, 2,5 a $3 \mathrm{~mm}$ de ancho. Las legumbres son de 3 a $6 \mathrm{~cm}$ de largo y de 1 a $2 \mathrm{~cm}$ de ancho, planas y carnosas, con el margen irregularmente enroscado o retorcido, con varias semillas; color café, lustrosas, comprimidas, elíptico-oblongas. Se multiplica por las semillas. Prospera en zonas cálidas (más de $30^{\circ} \mathrm{C}$ ), secas y con abundante exposición solar, cerca al mar a altitud menor de 250msnm (Programa de investigación aplicada a la medicina tradicional y popular del Caribe (TRAMIL)).

\section{Compuestos polifenólicos y su clasificación}

Los compuestos polifenólicos son metabolitos secundarios de las plantas que poseen en su estructura al menos un anillo aromático al que está unido uno o más grupos hidroxilo. Se clasifican como ácidos fenólicos, flavonoides y taninos. Existen alrededor de 8.000 compuestos polifenólicos identificados y la mayoría de estos poseen una estructura de 3 anillos, dos aromáticos y uno heterociclo oxigenado. Los compuestos polifenólicos más sencillos poseen solo un anillo aromático y conforme aumenta el número de sustituyentes, se va incrementando la complejidad de la estructura. Previendo la gran diversidad de estructuras derivadas, se les ha agrupado en 12 familias.

Así, los flavonoides tienen dos anillos aromáticos unidos por una cadena de tres átomos de carbono $\left(\mathrm{C}_{6} \mathrm{C}_{3} \mathrm{C}_{6}\right)$. Por su parte, los taninos son compuestos poliméricos más complejos que se clasifican en hidrolizables y condensados. Los taninos hidrosolubles están constituidos por unidades de ácido elágico, y pueden estar unidos a una molécula 


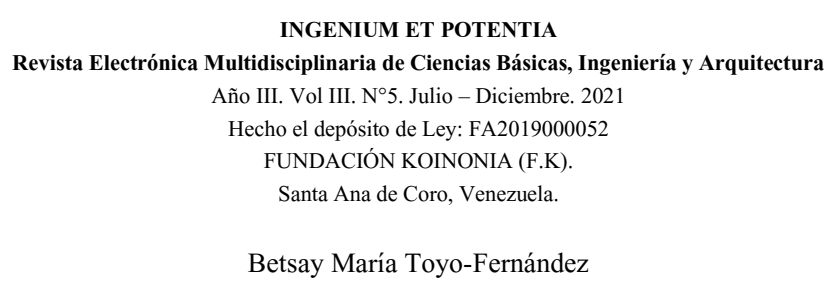

de glucosa. En cambio, los taninos condensados resultan de la condensación de unidades de flavan-3-oles, tales como la catequina que tienden a polimerizarse. Los compuestos polifenólicos son sustancias biológicamente activas y existen numerosas evidencias, epidemiológicas, estudios in vitro, estudios en modelos animales e intervenciones en humanos, que indican que estos compuestos proporcionan un beneficio al organismo en contra diversas enfermedades. Entre las propiedades benéficas de estos compuestos están la protección contra lesiones celulares y subcelulares, inhibición del crecimiento de tumores, activación de los sistemas de detoxificación hepáticos y bloqueo de las vías metabólicas que pueden ocasionar carcinogénesis (Mercado et al., 2013).

\section{Antioxidantes}

Los antioxidantes son moléculas capaces de retardar o prevenir la oxidación de otras moléculas a través de un mecanismo que suele conllevar su propia oxidación. La oxidación es una reacción química de transferencia de electrones de una sustancia a un agente oxidante. Estas reacciones pueden producir radicales libres que comienzan reacciones en cadena que dañan las células. Esto se produce debido a que los radicales libres son átomos o grupos de átomos que tienen un electrón desapareado con capacidad de aparearse, por lo que son muy reactivos, por lo tanto, recorren nuestro organismo intentando robar un electrón de las moléculas estables con el fin de alcanzar su estabilidad electroquímica y lograr su función específica en la célula (Burneo, 2009).

\section{Análisis de la actividad antioxidante por el ensayo del radical DPPH}

El radical 2,2-difenil-1-picrilhidrazilo (DPPH) fue uno de los primeros radicales sintéticos utilizados para estudiar la relación estructura-actividad de los antioxidantes fenólicos. El método se basa, en el descenso de absorbancia a $517 \mathrm{~nm}$, asociado a la desaparición de la forma radical del DPPH, de los medios de reacción provocada por antioxidantes (ver reacción 1). 
INGENIUM ET POTENTIA

Revista Electrónica Multidisciplinaria de Ciencias Básicas, Ingeniería y Arquitectura
Año III. Vol III. N5. Julio - Diciembre. 2021
Hecho el depósito de Ley: FA2019000052
FUNDACIÓN KOINONIA (F.K).
Santa Ana de Coro, Venezuela.
Betsay María Toyo-Fernández

$\mathrm{DPPH}^{\bullet}+\mathrm{AH} \rightarrow \mathrm{DPPH}-\mathrm{H}+\mathrm{A}^{\bullet} \quad$ Reacción 1

Se considera que el punto final del ensayo se alcanza cuando la absorbancia se mantiene constante. Por lo tanto, el tiempo de ensayo puede variar desde unos minutos, hasta algunas horas dependiendo del tipo y de las concentraciones de los diferentes antioxidantes presentes en las muestras analizadas (Frankel y Meyer, 2000).

\section{METODOLOGÍA}

\section{Método}

La combinación de técnicas y metodologías, producto de elecciones realizadas por el investigador, no es un suceso superficial, sino que se arraiga en la orientación teórica que sigue una investigación y que tiene alcances a nivel epistemológico y ontológico. En este sentido, la opción por los métodos mixtos abre la puerta a una ciencia reflexiva que se pregunte acerca de qué es lo que puede conocer, de qué forma lo hace y mediante qué instrumentos técnicos puede hacerlo (Cueto, 2020). Así mismo, Mendizábal (2018) expresa que, la utilización de los métodos mixtos implica la combinación de perspectivas teóricas, epistemológicas, puntos de vista y de métodos cualitativos y cuantitativos, logrando mayor comprensión de la problemática, confianza en los datos, posibilidad de corroborar los resultados, complementariedad de las perspectivas, desarrollo de otras técnicas, profundidad y amplitud, captación de procesos y correlaciones.

En relación a esto, Gheno (2010) describe que es importante aplicar un enfoque integral para el estudio de los recursos naturales en particular plantas medicinales de modo que, entendiendo la parte cultural, ecológica y utilitaria, puedan establecerse líneas de investigación ecológica, etnofarmacológica, fitoquímica y de validación de actividad biológica de los recursos vegetales.

Por lo antes expuesto, se aplicará el método etnográfico, pues este busca describir contextualmente las relaciones complejas entre prácticas y significados para unas 


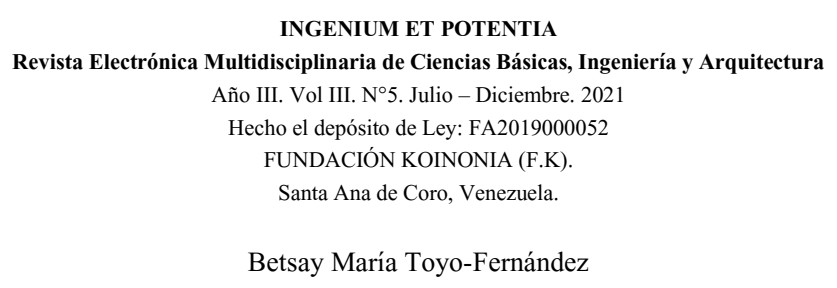

personas concretas sobre algo en particular (Restrepo, 2016). En este sentido, en la comunidad de Carazao perteneciente al Municipio Miranda del Estado Falcón se desarrollará el estudio, pues es lugar de residencia de la investigadora y esto permitirá un acercamiento y contacto a la comunidad y a sus prácticas etnomedicinales mucho más satisfactorio. El diseño del método etnográfico es realista o mixto (Hernández, Fernández y Baptista, 2014), pues se recolectarán datos tanto cualitativos como cuantitativos; estos últimos para el análisis de la importancia cultural del dividivi. Posterior a la aplicación del método etnográfico, prosigue la aplicación del método hipotéticodeductivo para determinar experimentalmente qué método y sistema es más adecuado para la extracción y definir si el contenido fenólico y capacidad antioxidante de los frutos secos de dividivi son la base de su acción terapéutica.

\section{Selección del área de investigación}

Se seguirá con los lineamientos establecidos por Rodríguez (2014) y Rodríguez et al., (2019); para ello, debe realizarse una descripción del área de investigación en cuanto a la ubicación de la comunidad, límites geográficos y condiciones climáticas. Así mismo, se ejecutarán recorridos por la zona de estudio para conocer el territorio, las condiciones ecológicas, sociales y económicas; así como la selección de los asentamientos poblacionales y zonas de interés para la ejecución del trabajo de campo.

\section{Estudio Etnobotánico}

Para evaluar el conocimiento etnobotánico se aplicará las técnicas de observación participante, diario de campo, informante y entrevistas estructuradas. Se realizarán preguntas aplicando un instrumento de recolección de datos basado en la encuesta estructurada del Programa de investigación aplicada a la medicina tradicional y popular del Caribe (TRAMIL) complementada con algunos ítems que presenta la guía metodológica para estudio etnobotánico de especies forestales en comunidades amazónicas y afines (Rodríguez et al., 2019). La selección de los informantes viene dada 


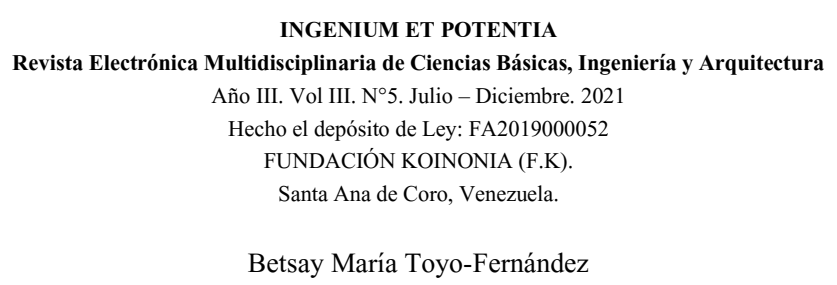

por el conocimiento de la investigadora sobre las personas de mayor edad que practican comúnmente la etnobotánica medicinal, aunado a la realización de consultas a líderes de los consejos comunales y el personal de salud del ambulatorio para que propongan a las personas con capacidades expertas en el conocimiento sobre el uso y manejo de las plantas medicinales nativas de la comunidad.

El total de los informantes claves estarán representados por hombres y mujeres de mayor edad en el grupo familiar (Bello, 2017; Bermúdez, 2002; Dávila et al., 2016 y Rodríguez et al., 2014) y se les solicitará que enlisten las plantas medicinales que conocen y/o usan, a fin de determinar si el dividivi Caesalpinia coriaria (Jacq.) Willd. surge espontáneamente en las listas, y en qué orden de aparición lo hace. En general, el formato de la encuesta registra los siguientes datos: información general del entrevistado, las plantas nativas de la localidad que conoce y utiliza; procedencia del conocimiento en plantas medicinales; frecuencia de uso de las plantas medicinales; partes de las plantas que se usan para elaborar los tratamientos; formas de preparación y administración, enfermedades para las que se recomiendan, dosificación para adultos y niños.

Además de las conversaciones y entrevistas recurrentes con los informantes, se realizará la observación de cómo los actores sociales aplican la etnobotánica medicinal del dividivi, es decir, desde la manera en cómo la recolectan, cómo preparan la medicina dependiendo de la enfermedad que quieran curar hasta la forma en cómo es administrada.

Se realizará la identificación de la especie en cada zona de muestreo mediante expediciones de colecta acompañados de los informantes claves para el registro in situ de los conocimientos etnobotánicos de la especie con potencial de uso. Las muestras serán llevadas hasta el herbario Coro-Departamento de investigación de la Universidad Politécnica Territorial Alonso Gamero (UPTAG) para su correcta determinación taxonómica (Rivas, 2015; Torres y Ganoza, 2017). 
INGENIUM ET POTENTIA

Revista Electrónica Multidisciplinaria de Ciencias Básicas, Ingeniería y Arquitectura
Año III. Vol III. N5. Julio - Diciembre. 2021
Hecho el depósito de Ley: FA2019000052
FUNDACIÓN KOINONIA (F.K).
Santa Ana de Coro, Venezuela.
Betsay María Toyo-Fernández

\section{Análisis cuantitativo}

De acuerdo con lo descrito por Molares et al., (2009) y Jaramillo et al., (2014), para el análisis de la importancia cultural del Dividivi (Caesalpinia coriaria (Jacq.) Willd.) se aplicarán los siguientes índices:

1. Índice de Friedman (IF) permite estimar la importancia relativa de cada especie a partir del grado de consenso de los informantes. Se calcula como: $I F=(I \mathrm{l} / \mathrm{lt}) \times 100$, donde: Ip: número de informantes que mencionaron una especie (frecuencia de mención); It: número total de informantes.

2. Índice de valor de uso (IUV): Expresa la importancia o valor cultural de una especie determinada para todos los informantes encuestados, estando referido a dos índices:

2.1. Índice de valor de uso para cada informante individual (IVUis), el cual considera que el valor de uso de cada una de las especies/informante está definido como la relación entre el número de usos mencionados en cada entrevista y el número de entrevistas realizadas por especie. Se calcula como: IVUis $=\Sigma U i s / n i s$, donde: Uis: número de usos mencionados por el informante i para la especie s en cada entrevista.; nis: número de entrevistas de dicho informante para una especie dada.

2.2. Índice de valor de uso general de cada especie para todos los informantes (IVUs), que representa el valor de uso promedio por informante de cada una de las especies consideradas. Se calcula como: IVUs= $\sum$ IVUis/ns, donde: ns: número de informantes entrevistados.

3. Consenso del uso i. Se calcula como: Consenso del uso $i=$ (número de entrevistados que informan sobre el uso i/número total de informantes que mencionan la planta)x100. 
INGENIUM ET POTENTIA

Revista Electrónica Multidisciplinaria de Ciencias Básicas, Ingeniería y Arquitectura
Año III. Vol III. N5. Julio - Diciembre. 2021
Hecho el depósito de Ley: FA2019000052
FUNDACIÓN KOINONIA (F.K).
Santa Ana de Coro, Venezuela.
Betsay María Toyo-Fernández

\section{Estudio fitoquímico}

\section{Obtención del material vegetal}

Los frutos del dividivi se recolectaran manualmente en época seca antes de las ocho de la mañana en cinco sectores distintos de la comunidad de Carazao, estado Falcón (Rivas, 2015).

\section{Preparación de los frutos del dividivi}

Los frutos se separarán de los materiales extraños, se secarán a la sombra a temperatura ambiente por espacio de ocho días, se pesarán y luego se colocarán en envases ámbar libre de humedad hasta su procesamiento a temperatura ambiente. De cada punto se tomarán de entre 5 a 10 plantas obteniéndose un aproximado de $5 \mathrm{Kg}$ de frutos secos de dividivi.

\section{Preparación de los extractos}

En la determinación del contenido fenólico y actividad antioxidante del material vegetal, las investigaciones varían en la clase de solventes utilizados y los métodos de extracción de los metabolitos secundarios. Por esta razón, en este estudio se utilizarán cuatro métodos de extracción para determinar si existen diferencias significativas entre cada método y cuál resulta ser el solvente más adecuado (hidroalcohólico o acuoso).

Así pues, el material vegetal se triturará en un mortero hasta obtener partículas finas, los extractos serán preparados al 10\% P/V usando cuatro sistemas: uno acuoso y tres etanolagua $(75-25 \%, 50-50 \%, 25-75 \%)$ a los que se les aplicarán cuatro métodos de extracción: soxhlet, maceración pasiva, infusión y decocción. La extracción se realizará por un período de 4 horas manteniendo un intervalo de temperatura de $60-70^{\circ} \mathrm{C}$ para el método soxlhet; la maceración pasiva se realizará a temperatura ambiente por 4 horas (Rivas, 2015). Por su parte, para preparar los extractos por decocción se le adicionará a cada sistema definido una porción del polvo del material vegetal y se llevará a ebullición; 


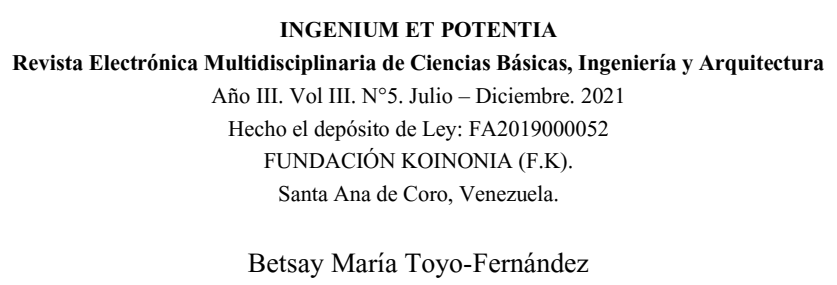

mientras que para la infusión se adicionará a otra porción del polvo a cada sistema caliente y se dejará reposar (Torres y Ganoza, 2017).

Los extractos concentrados se obtendrán por remoción al vacío del solvente en un rotoevaporador a $45-60^{\circ} \mathrm{C}$ dependiendo de la naturaleza del solvente. Se almacenarán en envases ámbar bajo condiciones de refrigeración. A cada extracto obtenido se le determinará los parámetros fisicoquímicos de humedad (método de peso constante), cenizas (norma COVENIN 1783-81), densidad (método empírico masa sobre volumen), índice de acidez (norma COVENIN 325:2001) e índice de refracción y el rendimiento de cada extracto siguiendo la norma COVENIN 1562-90, esto con el objeto de establecer qué sistema es el más adecuado considerando calidad y factibilidad del producto final (Rivas, 2015).

\section{Determinación de polifenoles totales}

El análisis de compuestos fenólicos totales se realizará por el método espectrofotométrico de follinciocalteu usando ácido gálico como patrón de acuerdo a la metodología descrita por Magalhaes et al., (2010), con algunas modificaciones (Rivas, 2015). A 200 $\mu \mathrm{L}$ de muestra se le adicionarán $1500 \mu \mathrm{L}$ de agua destilada, $100 \mu \mathrm{L}$ de reactivo follin y $200 \mu \mathrm{L}$ de carbonato de sodio al $7,5 \%$. La mezcla se sónica por 5 min a $50^{\circ} \mathrm{C}$. Se incubará en la oscuridad por 2 horas y se realizará la lectura a $790 \mathrm{~nm}$. La concentración de polifenoles se expresará como mg equivalentes de ácido gálico por g de dividivi seco.

\section{Actividad antioxidante}

La determinación del poder antioxidante de los extractos, estimada a través de su capacidad para desactivar radicales libres, se llevará a cabo siguiendo el método DPPH (depleción del óxido 2,2-difenil-1-picrilhydracilo) descrito por Gaviria et al., (2007) (citado por Rivas, 2015). Se preparará una solución de DPPH 20mg/L en etanol: agua, 1:2. Una alícuota de $980 \mu \mathrm{L}$ de esta solución se incubará a temperatura ambiente durante 2 horas y se medirá la absorbancia inicial a $517 \mathrm{~nm}$. Para la medición se mezclaron $20 \mu \mathrm{l}$ de los 


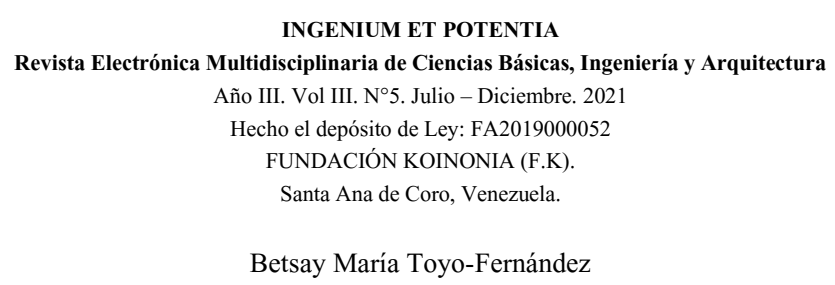

extractos con $980 \mu$ de la disolución (DPPH). La cuantificación de la actividad atrapadora de DPPH se llevará a cabo por sustracción de los valores de absorbancia a 517nm inicial del reactivo DPPH y de las muestras, considerando el descenso de absorbancia debido a la reducción del radical DPPH. Los resultados finales se expresarán como equivalentes mM TROLOX por mg de extracto de dividivi.

\section{CONCLUSIONES}

Los estudios etnobotánicos desde un enfoque interdisciplinario contribuyen a la búsqueda de nuevos agentes fitoterapéuticos y al desarrollo sostenible para las comunidades rurales portadoras del conocimiento tradicional.

El registro del conocimiento tradicional es fundamental, por ello la aplicación de la encuesta TRAMIL ha sido ampliamente utilizada; sin embargo, con el fin de completar y detallar la información etnobotánica en cuanto a: forma en que se obtuvo el conocimiento, parte de la planta utilizada, usos adicionales (además del medicinal), opciones en la forma de uso del remedio, localización de la planta y la asociación con otras plantas para la cura de la enfermedad se utiliza la encuesta de Rodríguez et al., (2019). El total de los informantes claves estarán representados por hombres y mujeres de mayor edad en el grupo familiar (Bello, 2017; Bermúdez, 2002; Dávila et al., 2016 y Rodríguez et al., 2014). Las determinaciones cuantitativas en los estudios etnobotánicos expresan la importancia cultural de la especie en ese contexto. Por ello, se determinará el índice de Friedman (IF), índice de valor de uso (que se expresa de dos maneras: índice de valor de uso para cada informante individual (IVUis) e Índice de valor de uso general de cada especie para todos los informantes (IVUs)) y consenso del uso (i) de acuerdo con lo descrito por Molares et al., (2009) y Jaramillo et al., (2014).

Para la realización del estudio fitoquímico de la especie deben prepararse extractos, generalmente son hidroalcohólicos (Rivas, 2015; Torres y Ganoza, 2017); por esto, se pretende obtener extractos utilizando mezclas etanol-agua al: $75-25 \%, 50-50 \%, 25-75 \%$ 
y uno acuoso (ya que por decocción es la forma como tradicionalmente se aplican los remedios de dividivi para las distintas afecciones).

La determinación del contenido fenólico se realizará por el método espectrofotométrico de follinciocalteu usando ácido gálico como patrón de acuerdo a la metodología descrita por Magalhaes et al., (2010), con algunas modificaciones (Rivas, 2015). Asimismo, la actividad antioxidante de los extractos de dividivi, estimada a través de su capacidad para desactivar radicales libres, se llevará a cabo siguiendo el método DPPH descrito por Gaviria et al., (2007) (citado por Rivas, 2015).

\section{FINANCIAMIENTO}

No monetario.

\section{AGRADECIMIENTO}

A todos los agentes involucrados en el desarrollo de la investigación.

\section{REFERENCIAS}

Adams, M.; Berset, C.; Kessler M. y Hamburguer, M. (2009). Plantas Medicinales para el tratamiento de los trastornos reumáticos. Una visión general de herbals europeos de los siglos XVI y XVII. Journal Ethnopharmacol, 121: p.343-359.

Alvarez, C. y Lock, O. (1992). Taninos. Revista de Química. VI (1): 47- 63.

Amudha, P. y Shanthi, P. (2011). Antioxidant activity of some rare medicinal plants. Journal of Pharmacy Research, 4(3):698-699.

Bello, J. (2017). Plantas medicinales silvestres y/o naturalizadas en la península de Araya, estado Sucre, Venezuela. Revista Saber, Universidad de Oriente, Venezuela, 29: 326-339.

Bermúdez, A. y Velásquez, D. (2002). Etnobotánica médica de una comunidad campesina del estado Trujillo, Venezuela: un estudio preliminar usando técnicas cuantitativas. Revista de la Facultad de Farmacia, 44: 2-6. 
INGENIUM ET POTENTIA

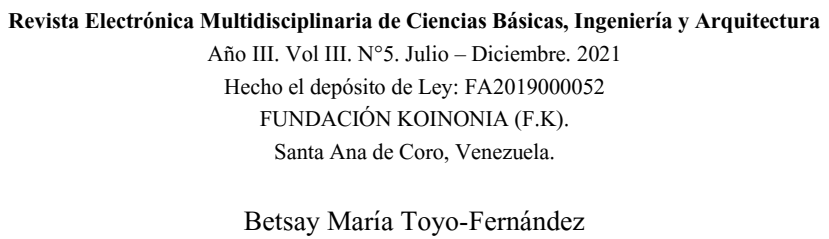

Bermúdez, A.; Oliveira, María. A. y Velázquez, D. (2005). La investigación etnobotánica sobre plantas medicinales: una revisión de sus objetivos y enfoques actuales. Revista Interciencia, 30 (8): 453-459.

Burneo, Z. (2009). Determinación del contenido de compuestos fenólicos totales y actividad antioxidante de los extractos totales de doce especies vegetales nativas de Sur del Ecuador (tesis de pregrado). Universidad Técnica Particular de Loja, Ecuador.

Cueto, J. (2020). Apuntes para la investigación mixta en antropología. Un caso de aplicación desde la etnografía. Cuadernos del Instituto Nacional de Antropología y Pensamiento Latinoamericano - Series Especiales, 8 (1): 97-111.

Dávila, M.; Pomboza, P.; Vásquez, C. y Gómez, T. (2016). Etnobotánica de plantas nativas de una comunidad rural de la Sierra, Ecuador: un análisis cuantitativo, Revista Arnaldoa, 23 (1): $219-234$.

Díaz, M. (2001). Ecología experimental y ecofisiología: bases para el uso sostenible de los recursos naturales de las zonas áridas neo-tropicales. Revista Interciencia, 26 (10), 472-478. Recuperado de: https://www.redalyc.org/articulo.oa?id=33906109

Frankel, E. y Meyer, A. (2000). The problems of using one-dimensional methods to evaluate multifunctional food and biological antioxidants. Journal Sci. Food Agric. 80: 1925-194.

Gaviria, C.; Cifuentes, O.; Monsalves, C. y Rojano, B. (2007). Actividad antioxidante de estractosmetanolicos de Attalea Butyracea. Revista Scientia Et Technica XIII 33: 297-298.

Gheno, y. (2010). La etnobotánica y la agrodiversidad como herramientas para la conservación y el Manejo de recursos naturales: un caso de estudio en la organización de parteras y médicos indígenas tradicionales 'nahuatlxihuitt' de ixhuatlancillo, veracruz, México (tesis de postgrado). Universidad Autónoma del Estado de México. Toluca, Estado de México.

Hernández, R., Fernández, C. y Baptista, M. (2014). Metodología de la investigación. McGraw-Hill. México, D.F, México. ISBN: 978-1-4562-2396-0. 600p 
INGENIUM ET POTENTIA

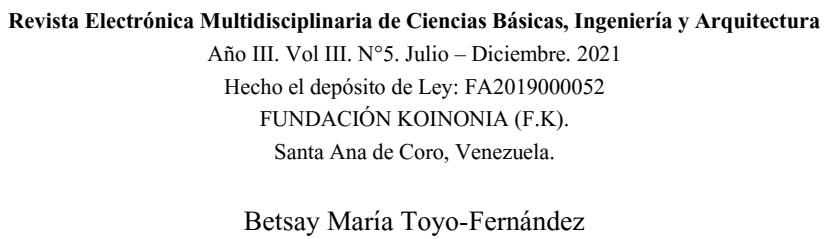

Hernández, V.; Mora, F. y Meléndez P. (2012). A study of medicinal plant species and their ethnomedicinal values in Caparo Barinas, Venezuela. Emirates Journal of Food and agriculture, 24 (2): 128-132. Retrieved from. Recuperado de: http://search.proquest.com.

Jaramillo, M.; Castro, M., Ruiz, T.; Lastres, M.; Torrecilla, P.; Lapp, M.; Hernández, L. y Muñoz, D. (2014). Estudio etnobotánico de plantas medicinales en la comunidad campesina de Pelelojo, municipio Urdaneta, estado Aragua, Venezuela. Revista Ernstia, 24 (1): 85-110. Recuperado de: https://www.researchgate.net/publication/279531479

Magalhaes, L.; Santos, F.; Segundo, M.; Reis, S. y Lima J. (2010). Rapid microplate highttroughput methodology for assessment of follin-ciocalteu reducing capacity. Journal Talanta, 83:441-447.

Mendizábal, N. (2018).La osadía en la investigación: el uso de los Métodos Mixtos en las ciencias sociales. Revista Espacio abierto, 27 (2) (abril - junio, 2018): 5-20.

Mercado, G.; Carrillo, L.; Wall, A.; López, J. y Álvarez, E. (2013). Compuestos polifenólicos y capacidad antioxidante de especias típicas consumidas en México. Revista Nutrición Hospitalaria, 28(1):36-46.

Molares, S.; González, S.; Ladio, A. y Castro, M. (2009). Etnobotánica, anatomía y caracterización físico-química del aceite esencial de Baccharis obovata Hook. et Arn. (Asteraceae: Astereae). Acta bot. bras. 23(2): 578-589. Recuperado de: http://www.botanica.org.br/acta/ojs

Plan de la Patria 2025. Proyecto Nacional Simón Bolívar, Tercer Plan Socialista de Desarrollo Económico y Social de la Nación, 2019-2025. Gaceta Oficial de la República Bolivariana de Venezuela № 6.446 Extraordinario. Caracas, lunes 8 de abril de 2019.

Programa de investigación aplicada a la medicina tradicional y popular del Caribe. Caesalpinea coriara. Recuperado de: http://www.tramil.net/es/plant/caesalpiniacoriara.

Restrepo, E. (2016). Etnografía: alcances, técnicas y éticas. Envión editores. Departamento de Estudios Culturales. Pontificia Universidad Javeriana. Bogotá, Colombia. ISBN: 978-958-99438-4-7. 100p 
INGENIUM ET POTENTIA

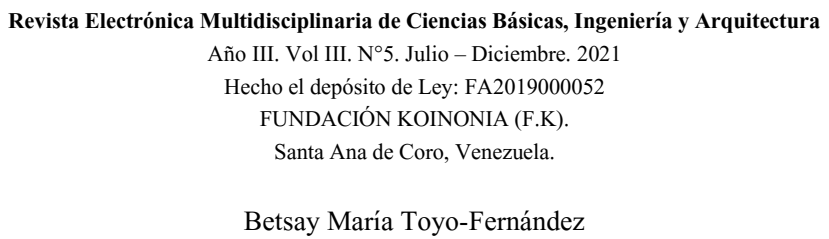

Rivas, B. (2015). Contenido de compuestos fenólicos y actividad antioxidante de extractos de orégano (Oreganum vulgare $L$ ) (tesis de postgrado). Universidad del Zulia, Maracaibo, Venezuela.

Rodríguez, Y. (2014). Etnobotánica, diversidad, fitoquímica y conservación de especies de interés medicinal en el parque nacional viñales (tesis de postgrado). Universidad de Pinar del Rio "Hermanos Saiz Montes de Oca", Pinar del Rio, Cuba.

Rodríguez, Y.; Valdés, M.; Hernández, H. y Soria, S. (2019).Guía metodológica para estudio etnobotánico de especies forestales en comunidades amazónicas y afines. Revista Cubana de Ciencias Forestales, 7(1):98-110. Recuperado de: http://cfores.upr.edu.cu/index.php/cfores/article/view/368

Rosado, J. y Moreno, M. (2010). Farmacopea guajira: el uso de las plantas medicinales xerofíticas por la etnia wayuu. Universidad de La Guajira, Riohacha-La Guajira, Colombia.

Rosas, R. (2015). Contribución al conocimiento etnofarmacobiólogico de plantas medicinales de la región Cañada, Oaxaca. Revista Iberoamericana de Producción Académica y Gestión Educativa. Universidad de la Cañada.

Sánchez, J.; Alvarez, L.; Marquina, S.; Salas, E.; Cuevas, V.; Jiménez, E.; Veloz, R.; Carraz, M. y González, L. (2017). Phenolic Compounds Isolated from Caesalpinia coriaria Induce S and G2/M Phase Cell Cycle Arrest Differentially and Trigger Cell Death by Interfering with Microtubule Dynamics in Cancer Cell Lines. Journal Molecules, 22, 666; doi:10.3390/molecules22040666

Sarmiento, D.; Espitia, L. y López, R. (2017). Caracterización de los Productos Forestales No Maderables del bosque seco tropical asociado a las comunidades del Caribe colombiano. Revista Brasileira de Biociências, 15 (4): 187-198

Torres, F. y Ganoza, M. (2017). Etnobotánica y sistemas de extracción para compuestos fenólicos, actividad antioxidante y toxicidad de plantas de páramos y bosques nublados del norte peruano. Revista peruana de medicina Integrativa, 2(2):101109.

Vargas, A. y Lobato, C. (2018). La etnobotánica como base para la obtención de nuevos fármacos. Revista de divulgación científica y tecnológica de la Universidad Autónoma de Nuevo León, 21 (88). 
INGENIUM ET POTENTIA

Revista Electrónica Multidisciplinaria de Ciencias Básicas, Ingeniería y Arquitectura

Año III. Vol III. N5. Julio - Diciembre. 2021

Hecho el depósito de Ley: FA2019000052

FUNDACIÓN KOINONIA (F.K)

Santa Ana de Coro, Venezuela.

Betsay María Toyo-Fernández

@2021 por el autor. Este artículo es de acceso abierto y distribuido según los términos y condiciones de la licencia Creative Commons Atribución-NoComercial-Compartirlgual 4.0 Internacional (CC BY-NC-SA 4.0) (https://creativecommons.org/licenses/by-nc-sa/4.0/). 